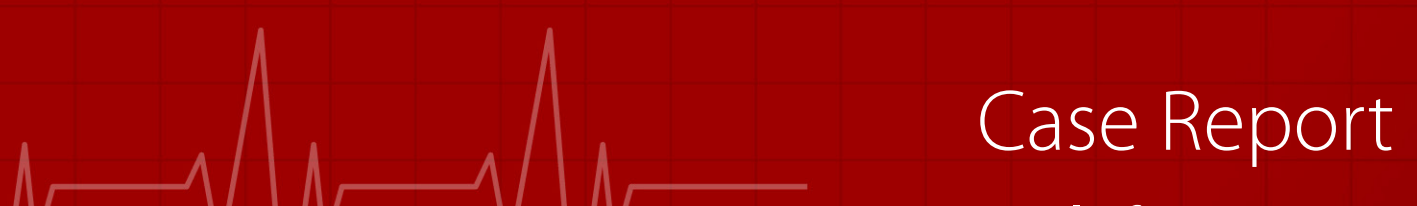

Journal of Emergency Medicine Case Reports

\title{
COVID-19 at First Glance, Pulmonary Tuberculosis With a Glance in Depth
}

Oğuz Abdullah Uyaroğlu', Nursel Çalık Başaran', Lale Özışı1', Ahmet Çağkan İnkaya², Gülay Sain Guven

'Hacettepe University Faculty of Medicine, Department of Internal Medicine, Ankara, Turkey

${ }^{2}$ Hacettepe University Faculty of Medicine, Department of Infectious Diseases and Clinical Microbiology, Ankara, Turkey

\section{Abstract}

Introduction: Here we report a case who presented with fever, dry cough, sore throat and myalgia, mimicking COVID-19 and diagnosed as Pulmonary Tuberculosis. Case Report: A 31-year-old female patient was presented to the COVID-19 outpatient clinic with 7-day history of fever,dry cough,night sweats,chills,sore throat,and myalgia. Laboratory results revealed lymphopenia, C-reactive protein, erythrocyte sedimentation rate and D-dimer elevation compitable with COVID-19. She was hospitalized with diagnosis of "probable COVID-19". SARS-CoV-2 multiplex PCR were resulted negative. Chest CT revealed cavitary lesion located in the upper lobe of the right lung with uncertain borders, consolidation in the right upper lobe primarily in favor of TB infection. She had three samples of sputum acid-fast bacillus smear that came back positive. TB PCR also resulted positive. Conclusion: Although the most common presentation of COVID-19 seems to be pneumonia, there is no unique clinical feature that reliably differentiates COVID-19 from other upper / lower airway viral or bacterial infections.

Keywords: Pulmonary Tuberculosis, COVID-19, SARS-CoV-2

\section{Introduction}

“Coronavirus Disease 2019” (COVID-19), caused by the virus, originally called 2019-nCoV, later named "SARSCoV-2" was declared as a pandemic by the World Health Organization (WHO) in March 2020 . Although the most common presentation of infection seems to be pneumonia, there is no unique clinical feature that reliably differentiates COVID-19 from other upper / lower airway viral infections. The most commonly documented reason for hospitalization is new onset cough and respiratory distress which are also main symptoms of pulmonary Tuberculosis (TB) ${ }^{2}$.

In 2018, an estimated 10 million people fell ill with TB worldwide $^{3}$. In Turkey the incidence of TB decreased from 29.4 per hundred thousand in 2005 to 14.1 per hundred thousand in $2018^{4}$. However TB should always be in the differential diagnosis of patients presenting with fever, cough, night sweats, and fatigue.

In the midst of pandemic, COVID-19 ranks the first in differential diagnosis of all patients with fever and respiratory symptoms. Physicians awareness is the cornerstone in differential diagnosis of different diseases presenting with similar symptoms.

Here we report a case who presented with fever, dry cough, sore throat and myalgia, mimicking COVID-19 and diagnosed as TB.

\section{Case Report}

A 31-year-old female patient presented to the COVID-19 outpatient clinic with 7-day history of fever, dry cough, night sweats, chills, sore throat, and myalgia. She had no travel history or no contact with anyone who had traveled anywhere or had COVID-19. Her body temperature was $38,5^{\circ} \mathrm{C}$. The rest of physical examination was unremarkable. Chest radiography showed prominent bronchovascular branches and increased localized reticulonodular density in the right upper lobe (Figure 1).

Laboratory results were as follows: hemoglobin 10,7 gr/dL (11.7-15.5), white blood count $6.6 \times 10^{3} / \mu \mathrm{L}$ (4.1-11.2 $\left.\mathrm{x} 10^{3} / \mu \mathrm{L}\right)$ with $0.92 \times 10^{3} / \mu \mathrm{L}\left(1.2-3.6 \times 10^{3} / \mu \mathrm{L}\right)$ lymphocytes count. C-reactive protein (CRP) was $7.59 \mathrm{mg} / \mathrm{dl}$ (normal; $<0.1$ ), erythrocyte sedimentation rate (ESR) was $80 \mathrm{~mm} / \mathrm{h}$ (0-25 mm/h). D-dimer was $1.81 \mathrm{mg} / \mathrm{L}(0-0.55)$ and ferritin was $47.5 \mu \mathrm{g} / \mathrm{L}$ (11-307).

She was hospitalized with diagnosis of "probable COVID-19 or TB" and isolated in a single room, with standard, contact and droplet precautions. Nasopharyngeal swab specimens were obtained for respiratory viral, bacterial and SARS-CoV-2 multiplex Real-Time Polymerase Chain Reaction (RT-PCR) test that all were resulted negative the next day. In the detailed history of the patient after she was hospitalized, it was learned that she has been coughing for 3 weeks and she unintentionally lost 2 kilograms during the same period. She had no sputum or hemoptysis. She noticed an increase in night sweats. Low dose chest computed to- 


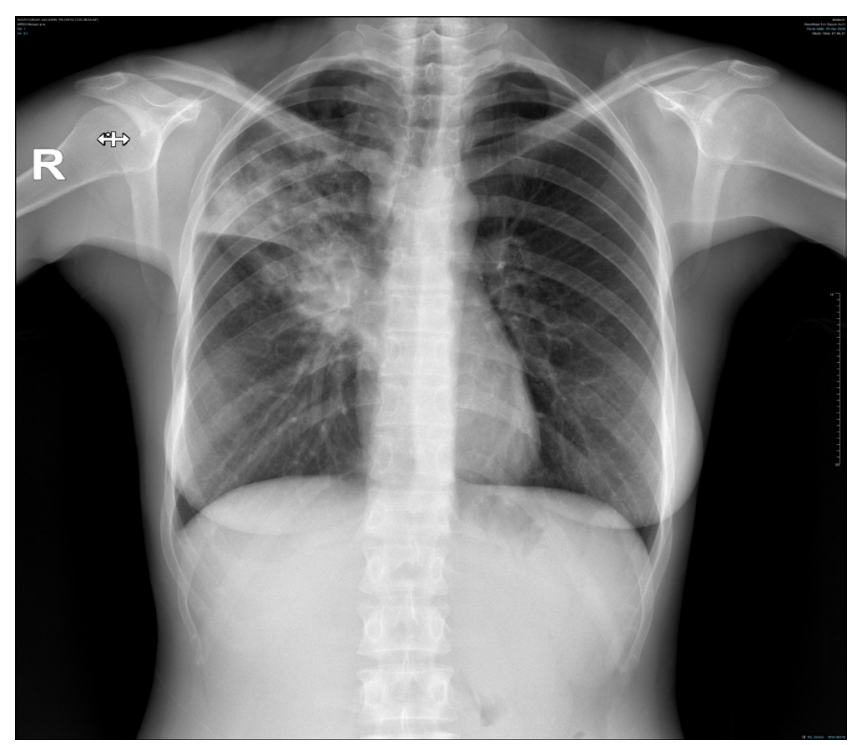

Figure 1: Postero-Anterior Chest Graph: Prominent bronchovascular branches and increased localized reticulonodular density in the right upper lobe

mography (CT) revealed cavitary lesion located in the upper lobe of the right lung with uncertain borders, consolidation in the right upper lobe primarily in favor of $\mathrm{TB}$ infection (Figure 2).

A bronchoalveolar lavage (BAL) sample was taken from the right upper lobe by fiberoptic bronchoscopy (FOB). She had three samples of sputum acid-fast bacillus (AFB) smear that came back positive. She had a skin purified protein derivative (PPD) test which showed active induration of 15 $\mathrm{mm}$. TB PCR also resulted positive. The patient started on four-drug regimen; isoniaside, rifampin, ethambutol and pyrazinamide.

On the 7th day of treatment, her fever resolved. The general condition of the patient improved. Laboratory values started to improve. The patient was discharged by a follow-up plan.

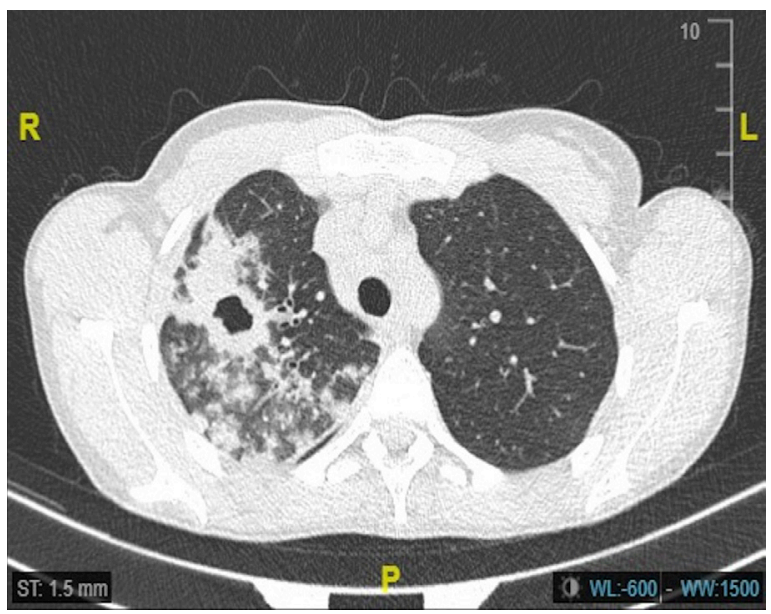

\section{Discussion}

The COVID-19 pandemic is an awe-inspiring situation. Although data regarding clinical presentations related to this new virus and the list of common symptoms is rapidly growing, fever and respiratory symptoms hold theirs' own.

TB and COVID-19 baseline clinics are similar. They are both transmitted mainly by airdrops with close contacts. They have similar symptoms such as fever, cough which could lead to delays in the timely diagnosis in the midst of pandemic. In fact TB is a chronic disease with cough of 2 weeks and COVID-19 has a rapid onset ${ }^{5}$, but it can be challenging to take a detailed history of symptoms with masks, glasses and PPE as it happened in our case.

COVID-19 and TB also have similar laboratory findings. Lymphopenia accompanied by mild thrombocytopenia is among the most common abnormal findings, attracting the attention of COVID-19 patients. The total white blood cell count or neutrophil counts are found to vary ${ }^{1}$. While lymphopenia and thrombocytopenia are rare in the course of TB, mostly normocytic anemia, leukocytosis, thrombocytosis, and elevated liver enzymes are seen as a result of increased cytokine activity ${ }^{6}$. Although anemia may be seen in COVID-19 patients at admission, it has been reported that this may be associated with chronic disease anemia due to underlying comorbidities rather than the pathophysiology of COVID-197 . ESR has been shown to be a prognostic factor in both COVID-19 and $\mathrm{TB}^{7,8}$. As expected, in inflammatory processes CRP and ferritin also increase during both COVID-19 and TB. While the procalcitonin (PCT) level is usually within the normal range at the initial diagnosis of COVID-19, it has been shown to increase in those with bacterial co-infection and intensive care patients ${ }^{7}$. PCT is also not generally elevated in pulmonary TB patients however when serum PCT is not within the normal range, it is a poor prognostic marker ${ }^{9}$. Increased levels of lactate dehydrogenase (LDH), alanine aminotransferase (ALT), aspartate ami-

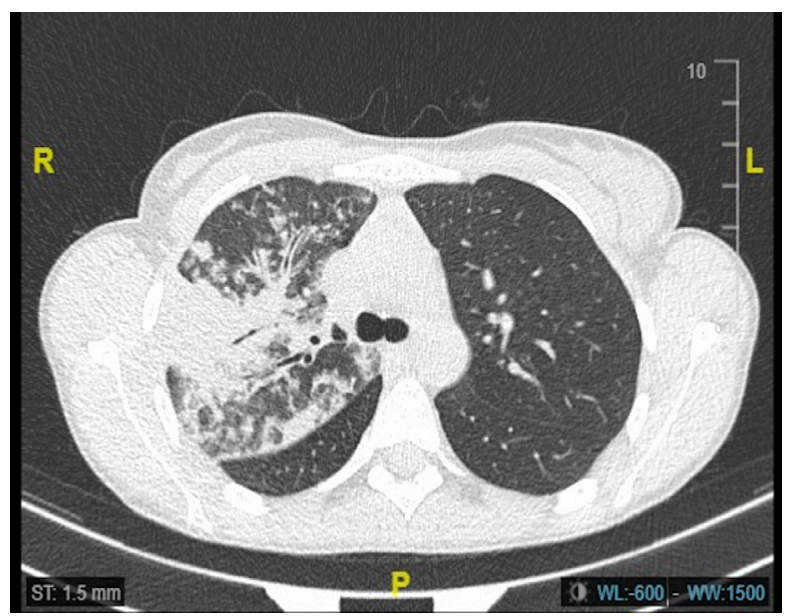

Figure 2: Chest CT: Cavitary lesion in the right lung with uncertain borders of the upper lobe, consolidation in the upper lobe anterior segment and distal-inferior cavitary lesion, patchy ground glasses in the middle lobe, patchy opacities that tend to merge in the upper lobe superior segment and adjacent major fissure area 
notransferase (AST) and decreased levels of albumin are among the most common abnormal biochemical laboratory findings in COVID-19 patients, and elevated D-dimer support the occurrence of coagulopathy as an important indicator of disease progression ${ }^{7}$. In our case, all these similar findings first suggested the diagnosis of COVID-19. However, TB should still be first in the differential diagnosis in patients presenting with anemia, elevated ESR, and CRP.

Pulmonary TB is caused by the bacterium Mycobacterium tuberculosis complex. The bacterium is easily spread from an infected person to someone else. Bacteria can be contracted by breathing in air droplets from a cough or sneeze of an infected person. The resulting lung infection is called primary TB. Most people recover from primary TB infection without further evidence of the disease. The infection may stay inactive (dormant) for years. In some people, it reactivates. Symptoms of pulmonary TB are cough, difficulty in breathing, excessive sweating (particularly at night), fever, fatigue, weight loss ${ }^{10}$. According to guidelines, the diagnosis of pulmonary TB should include smear and culture of sputum samples for ARB, as well as nucleic acid amplification testing ${ }^{11}$.

Diagnosis of COVID-19 is challenging for a couple of reasons: Firstly preferred diagnostic test for COVID-19 is a RT-PCR test that detects SARS-CoV-2 from the sample taken from the upper respiratory tract ${ }^{12}$. But RT-PCR sensitivity reported to be $63 \%$ for nasal swabs ${ }^{13}$. Secondly; diagnostic value of chest X-ray in COVID-19 pneumonia ranges from $30-60 \%$. CT plays an important role in the early stages of infection but it can not be easily available in all settings.

COVID-19 and TB are both infectious diseases that primarly involve the respiratory tract. They have very similar symptoms and laboratory findings which could lead to delays in the timely diagnosis in the midst of pandemic. COVID-19 is grabbing the headlines, but other diseases - including TB haven't stopped yet. Physicians' awareness is the cornerstone of the precise diagnosis of diseases with similar diagnostic tests, presenting with similar symptoms.

*Written consent has taken from our patient.

Acknowledgement: N/A

Conflict of Interest: The authors declare no potential conflicts of interest.

Funding: None of the authors received a financial support and/or specific funding for this work.

\section{References}

1. Wu Z, McGoogan JM. Characteristics of and Important Lessons From the Coronavirus Disease 2019 (COVID-19) Outbreak in China: Summary of a Report of 72314 Cases From the Chinese Center for Disease Control and Prevention. JAMA. 2020;323(13):1239-1242. doi:10.1001/ jama.2020.2648.

2. Huang, Chaolin, $X \mathrm{Li}$, et al. "Clinical features of patients infected with 2019 novel coronavirus in Wuhan, China." The lancet 395.10223 (2020): 497-506.

3. World Healt Organization. Global Tuberculosis Report 2019, Avaible from: https://www.who.int/tb/global-report-2019.

4. Bakanlığı, TC Sağlık. "Tüberküloz tanı ve tedavi rehberi." 2. Baskı Ankara, Mayıs 2019. Avaible from: https://hsgm.saglik.gov.tr/depo/birimler/tuberkuloz_db/haberler/Tuberkuloz_Tani_Ve_Tedavi_Rehberi_/Tuberkuloz_Tani_ve_Tedavi_ Rehberi_08.07.2019_Yuksek_KB.pdf.

5. Adepoju P. Tuberculosis and HIV responses threatened by COVID-19. Lancet HIV. 2020;7(5):e319-e320. doi:10.1016/ S2352-3018(20)30109-0.

6. Oliva, V. M., Cezário, G. A. G., Cocato, R. A., \& Marcondes-Machado, J.. (2008). Pulmonary tuberculosis: hematology, serum biochemistry and the relation with the disease duration. Journal of Venomous Animals and Toxins including Tropical Diseases, 14(1), 71-81.

7. Pourbagheri-Sigaroodi, Atieh, et al. "Laboratory findings in COVID-19 diagnosis and prognosis." Clinica Chimica $\operatorname{Acta}(2020)$.

8. Mandal, Saket Kumar, and Laxmikant Chavan. "Erythrocyte sedimentation rate values in cases of active tuberculosis without HIV co-infection." J Med Sci Clin Res 4.10 (2016): 13156-9.

9. Ugajin, Motoi, et al. "Usefulness of serum procalcitonin levels in pulmonary tuberculosis." European Respiratory Journal 37.2 (2011): 371-375.

10. Leung, Ann N. "Pulmonary tuberculosis: the essentials." Radiology 210.2 (1999): 307-322.

11. World Health Organization. "The use of loop-mediated isothermal amplification (TB-LAMP) for the diagnosis of pulmonary tuberculosis: policy guidance." (2016).

12. Information for Healthcare Professionals about Coronavirus (COVID-19). Updated June 1, 2020. Avaible from: https:// www.cdc.gov/coronavirus/2019-nCoV/hcp/index.html

13. Wang W, Xu Y, Gao R, et al. Detection of SARS-CoV-2 in Different Types of Clinical Specimens. JAMA.2020;323(18):18431844. doi:10.1001/jama.2020.3786. 